\title{
Leiomyosarcoma of Intrahepatic IVC - Treatment with Radical Resection and IVC Replacement with PTFE Graft: A Case Report and Review of Literature
}

\author{
Chaudhary RJ ${ }^{1}$, Rastogi $\mathbf{A}^{1}$, Pahari $\mathrm{H}^{1}$, \\ Thiagarajan S1, Bhangui P1, Goja S1, Gautam D' \\ Piplani $\mathbf{T}^{3}$ and Soin $\mathbf{A S}^{1}$ \\ ${ }^{1}$ Medanta Institute of Liver Transplantation and \\ Regenerative Medicine, India \\ ${ }^{2}$ Department of Pathology, Medanta - the Medicity, India \\ ${ }^{3}$ Department of Radiology, Medanta - the Medicity, India \\ *Corresponding author: Rohan J agat Chaudhary, \\ Medanta Institute of Liver Transplantation and \\ Regenerative Medicine, Gurugram, India
}

Received: March 23, 2017; Accepted: J une 15, 2017; Published: J une 22, 2017

\begin{abstract}
Leiomyosarcomas of the Inferior Vena Cava (IVC) are rare tumors accounting for only $0.5 \%$ of all soft tissue sarcomas in adults with fewer than 300 cases reported. Intraluminal leiomyosarcoma of IVC is rare. The involvement of renal or hepatic veins determines the strategy for vascular reconstruction. Recent studies have shown that radical surgery with adjuvant multimodal therapy improves the cumulative survival rate. We report a case of IVC leiomyosarcoma in a young healthy woman along with diagnostic workup followed by resection of tumor and reconstruction of IVC by Polytetrafluoroethylene (PTFE) graft. She reports no recurrence/complications after 6 cycles of chemotherapy and 40 months of follow-up. The prognosis depends on grade of the tumor, segment of the IVC involved (level 2-better prognosis), size of tumor (size $<9 \mathrm{~cm}$ better prognosis) and adjuvant therapy. However, overall leiomyosarcoma runs a poor prognosis; over half of the patients with surgical excision develop recurrence. The 5-year survival rate for these patients ranges between 31 and $62 \%$.
\end{abstract}

Keywords: Inferior vena cava; Leiomyosarcoma; PTFE (Polytetrafluoroethylene ); Vascular reconstruction

\section{Introduction}

Primary vascular leiomyosarcoma is a rare tumor of mesenchymal origin and arises from the smooth muscle cells of the tunica media predominantly within the inferior vena cava $[1,2]$. Extraluminal tumor growth along the adventitia of the Inferior Vena Cava (IVC) seems to be the common presentation [1,3]. Intraluminal tumor growth is rarely found. The origin of the tumor is described in relation to the hepatic and renal veins. The IVC is divided into three levels [1,4-6]: Level 1 extends from the entry of the hepatic veins up to the right atrium; Level 2 comprises the area between the confluences of the renal and hepatic veins; Level 3 includes the area below the renal veins.

Retroperitoneal tumors are often not diagnosed until the disease is at an advanced stage with large tumor growth and involvement of surrounding structures. This is partly because of the nonspecific clinical presentation as well as absence of early symptoms. Most patients present with abdominal or flank pain $[1,4]$.

Radical en bloc resection of the affected venous segment remains the only therapeutic option associated with prolonged survival $[1,4,5]$. Ito, et al. in a recent study with leiomyosarcoma of the IVC concluded that radical surgery combined with adjuvant multimodal therapy had a 5-year cumulative survival rate of $62 \%$ [6].

The goals of surgical management of these tumors include the achievement of local tumor control, maintenance of caval flow, and the prevention of recurrence. The involvement of renal or hepatic veins determines the strategy for vascular reconstruction.

Reconstruction of the IVC is not always required, because gradual occlusion of the IVC allows the development of venous collaterals. However, when pararenal leiomyosarcoma of the IVC is present, reconstruction of the IVC and the renal vein is necessary to prevent transient or permanent renal dysfunction $[1,5,7]$.

We report a case of leiomyosarcoma of intrahepatic IVC with discussion of the surgical procedure and reconstruction of caval continuity

\section{Case Presentation}

A 40 year old previously healthy female presented with abdominal pain and generalised discomfort over a span of 3 months. She was advised a routine USG abdomen which showed a retroperitoneal mass in right hypochochondrium, rest was normal. Her Complete Blood Count $(\mathrm{CBC})$ and basic metabolic panel were within normal limits. Tumor markers [Beta Human Chorionic Gonadotropin (HCG), Ca 125, Alfa Fetoprotein (AFP), Ca 19.9, Carcinoembryonic antigen (CEA)] were normal.

\section{Radiology workup}

Contrast Enhanced Computed Tomography (CECT) Abdomen revealed well-defined homogenous, hypodense mass measuring 9.9 $\mathrm{cm}$ (CC) X $5.5 \mathrm{~cm}$ (TR) X $6 \mathrm{~cm}$ (AP) in upper retro peritoneum on right side, in close relation to IVC in its intrahepatic course. The mass showed moderate post-contrast enhancement and no necrosis/fat density/calcification. The mass was abutting posterior wall of IVC with significant compression of its lumen in the intrahepatic course. Right adrenal gland appeared adherent to the mass. Hepatic venous confluence into IVC was normal. Right renal vein was not visualized and right kidney was cystic - dysplastic, non functional. Left renal 

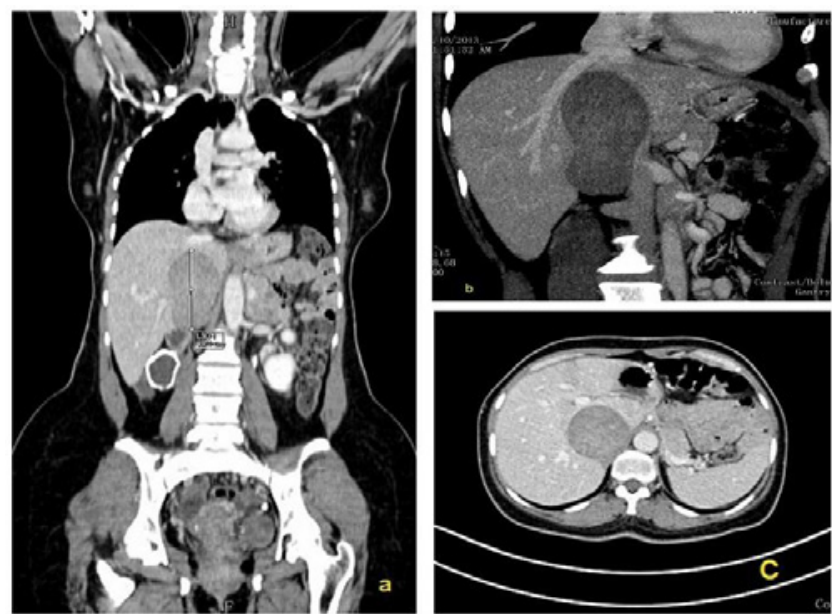

Figure 1: PET CT triphasic (a-c) revealed well-defined mass measuring 9.9 $\mathrm{cm} \times 5.5 \mathrm{~cm} \times 6 \mathrm{~cm}$ in upper retroperitoneum on right side, in close relation to IVC in its intrahepatic course. The mass showed moderate post-contrast enhancement. Hepatic venous confluence into IVC was normal. Right renal vein was not visualized and right kidney was cystic-dysplastic, non functional. Left renal vein was normal.

vein was normal. A differential diagnosis of either level 2 IVC tumor (leiomyoma or a low grade leiomyosarcoma), exophytic right adrenal mass or mesenchymal tumour of retroperitoneum was made.

A Positron Emission Tomography (PET) scan was performed from skull base to mid thigh with concurrently acquired triphasic CT for attenuation correction and anatomical localisation. It showed that the mass was non-FDG avid and there was no evidence of extrahepatic disease (Figure 1).

A diagnosis of retroperitoneal tumor was made and excision of the mass was planned if it was free of IVC. We would consider cross clamping of IVC if the mass was found arising from the wall of IVC and keep a backup for auto transplantation of liver. Cardiac surgery team was informed as a backup if required.

\section{Operative procedure}

Abdomen opened with a Mercedes Benz incision. There was no evidence of metastasis. The small intestine and large colon were mobilised. Rt kidney was shrunken. Liver was fully mobilized from the left and right sides. There was a heterogeneous vascular mass arising from intra-hepatic part of IVC between the renal and hepatic veins (level 2), extending more to the right, measuring $5 \times 9 \mathrm{~cm}$. The mass had compressed and distorted the IVC. All vascular communications between mass in IVC anteriorly and the caudate lobe were ligated and divided. Retro-hepatic cava was mobilized off the posterior abdominal wall. IVC above renal veins was looped which provided proximal control. IVC just below hepatic veins was looped which provided distal control. Segment of IVC $(10 \mathrm{~cm})$ with the mass was removed between vascular clamps. Frozen section of proximal and distal IVC margins were sent. With the tumor removed, a PTFE graft $22 \mathrm{~mm}$ in diameter and $10 \mathrm{~cm}$ in length was used as conduit and end to end anastomoses were performed using running 4-0 prolene at both ends (Figure 2). The IVC flow was restored. Frozen section of the IVC margins were negative for malignancy. Abdomen was closed after securing haemostasis.
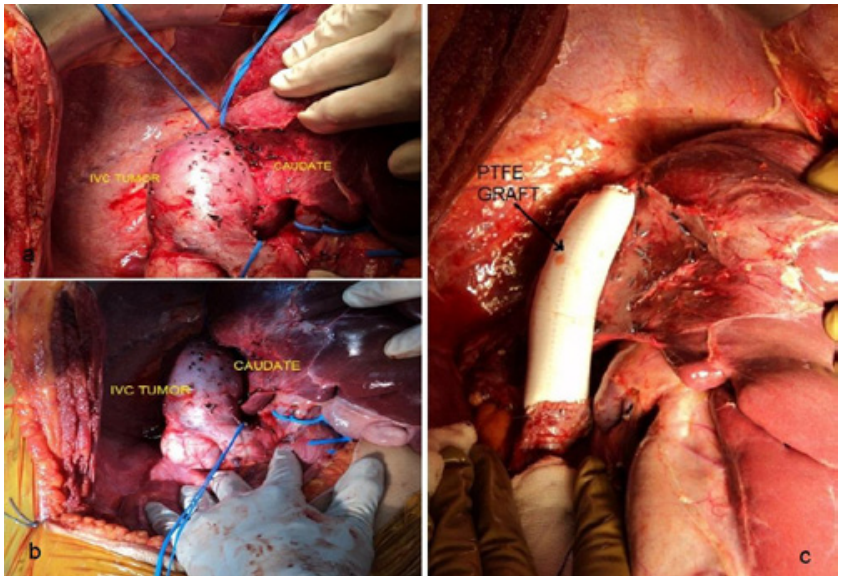

Figure 2: Operative procedure. a,b - Liver was mobilized from left \& right sides. There was a heterogeneous vascular mass arising from intra-hepatic part of IVC, extending more to the right, measuring $5 \times 7 \mathrm{~cm}$. The mass had compressed and distorted the IVC. All vascular communications between mass in IVC anteriorly and caudate were ligated \& divided. Posterior retrohepatic caval dissection was done. IVC above renal veins was looped which provided distal control. IVC just below hepatic veins was looped which provided proximal control. c - Segment of IVC $(10 \mathrm{~cm})$ with the mass was removed between vascular clamps. PTFE graft no 22 of adequate length $10 \mathrm{~cm}$ was used as conduit and an end to end anastomosis was completed using running 4-0 prolene.
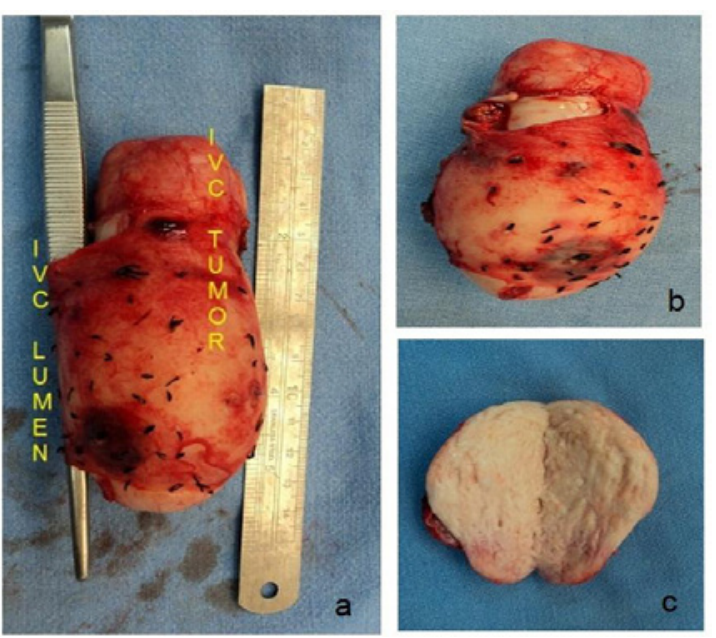

Figure 3: Specimen (a-c) - Grossly the specimen was globular soft tissue mass measuring $95 \times 70 \times 45 \mathrm{~mm}$. Cut surface showed grey white, firm and had whorled appearance.

On Histopathological examination, Grossly the specimen was globular soft tissue mass measuring $9.5 \mathrm{~cm}$. Cut surface showed grey white, firm and had whorled appearance. Outer surface inked red. It weighed 190 grams (Figure 3). On microscopy, section showed an encapsulated tumour composed of spindle cells seen in intersecting fascicles. Focal herring bone pattern was seen. The cells showed moderate pleomorphism with focal bizarre nuclei, tumour giant cells. Mitosis was increased (30-35/50 hpf). Resection margins were free. On IHC, SMA was diffusely positive, S-100 was negative, Ki67 was $15-20 \%$. Further IHC markers were performed on request. Vimentin was positive, CD117 was negative. Desmin was positive. Caldesmon was positive. CD 10 was negative. The final impression 


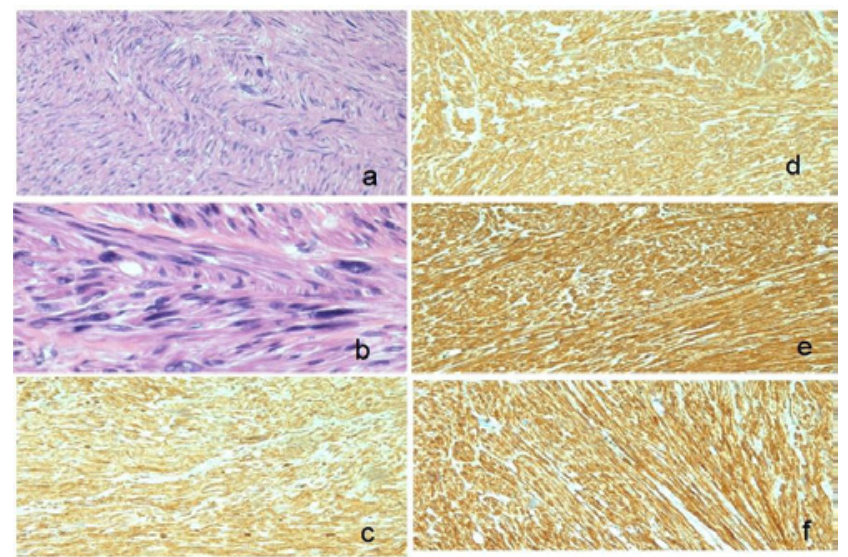

Figure 4: Histology - The final impression was Leiomyosarcoma of intrahepatic IVC; High grade. a,b - On Haematoxylin and eosin (10x, 40x), section showed an encapsulated tumour composed of spindle cells seen in intersecting fascicles. Focal herring bone pattern was seen. The cells showed moderate pleomorphism with focal bizarre nuclei, tumour giant cells. Mitosis was increased (30-35/50hpf). However, many atypical mitosis are seen.

c- Vimentin was positive, d- Caldesmon was postive, e- SMA was diffusely postive, f- Desmin was positive.

was Leiomyosarcoma of intrahepatic IVC; High grade (Figure 4).

The patient had an unremarkable postoperative course and was discharged on postoperative day (POD) 7. Postoperatively patient received anticoagulation with intravenous heparin for 5 days and then long-term oral anticoagulation to achieve target INR of 2.5. Patient received Deep Vein Thrombosis (DVT) prophylaxis measures. Daily ultrasound dopplers were done to confirm IVC patency.

Patient was followed up with monthly Doppler ultrasound to confirm IVC patency for 3 months. Patient received 2 cycles of MAID (Mesna, Doxorubicin, Ifosfamide, Dacarbazine) and 4 cycles of Docetaxel and Gemcitabine over six months. The patient tolerated the adjuvant therapy well. Presently, she is without clinical evidence of disease after 40 months of follow up and leading a normal life. Recent CT triphasic Abdomen and pelvis showed a patent IVC and no recurrence of disease.

\section{Discussion}

\section{Pathologic features}

Pathological features of the IVC leiomyosarcoma help in understanding clinical and radiological features better. In majority of retroperitoneal leiomyosarcomas growth is extrinsic to blood vessels. Tumors expand along tissue planes of least resistance often displacing adjacent organs. Direct invasion of the viscera is rare at least initially. The tumor is circumscribed by a pseudocapsule formed when the expanding tumor compresses adjacent organs. Intraluminal leiomyosarcomas are smaller than those that are extravascular. Often they present as nodular or polypoid masses attached to the vessel wall firmly. The cut section has a whitish gray whorled appearance. The intraluminal growth expands the IVC diameter, at times extending into the right heart and even into the pulmonary artery. Leiomyosarcomas with extraluminal and intraluminal growth pattern show great variation in the size of individual components. The finding of intra and extraluminal growth pattern is unique to leiomyosarcoma compared to other primary retroperitoneal tumors
[3].

Leiomyosarcoma on a microscopic level present a typical pattern of interlacing bundles of spindle shaped cells with blunt ended nuclei. Metastases to the liver, lungs and lymph nodes usually occur late. Recurrences are common, often exhibiting more aggressive histologic features than the original neoplasm [3].

\section{Clinical Presentation}

Leiomyosarcoma of the IVC are often limited in clinical expression. The main presenting symptoms are abdominal pain (as was the case in our patient), a palpable mass and lower extremity edema. Symptoms vary according to dimension of the tumor, growth pattern and localization of the tumor [8-10].

Suprahepatic leiomyosarcoma generally present with intraluminal venous extension and may result in Budd Chiari syndrome with hepatomegaly, jaundice and massive ascites [7]. The right heart and pulmonary artery may be involved. Nausea, vomiting and lower extremity edema may be present. Middle segment tumors may present with right hypochondrium and epigastric pain simulating hepatobiliary disease. Extension to suprahepatic veins may present with symptoms of Budd Chiari syndrome, while renal dysfunction results from extension into renal vessels [11-13]. Tumors of the lower segment of the IVC, which usually grow extraluminally can present with hypogastric and right iliac fossa pain $[11,13]$. Lower extremity edema is common.

\section{Diagnosis}

Noninvasive imaging modalities such as CT scan, USG and MRI are often used to diagnose these tumors. They provide valuable information regarding their origin, evaluating the presence of local invasion and excluding distant metastases. The sensitivity and specificity of CT in assessment of the tumors are 78\% and 96\% respectively and even higher with MRI (95-100\%) [14]. Ascending cavography can delineate the involvement of renal and hepatic veins and allow biopsy of the tumor. Selective arteriography of the celiac trunk may also be performed in patients where hepatic invasion or metastasis is suspected and transesophageal echocardiography can exclude or verify intracardial tumor extension [14]. Percutaneous venous biopsy with $\mathrm{CT}$ or echo guidance provides pathological confirmation.

\section{Operative management}

Resection of the retro/intrahepatic IVC is a technically demanding procedure but can be useful to allow extension of a potentially curable resection. Clinical conditions requiring resection of the inferior vena cava (IVC) are rare. Traumatic or iatrogenic injury, chronic post-thrombotic or membranous occlusion, and malignancy are the main indications. The notable malignant conditions include renal cell carcinoma, Wilms tumor, leiomyosarcoma, adrenal tumor, hepatic carcinoma, and retroperitoneal metastatic lymph nodes from testicular carcinoma [7].

Operative management for IVC tumors (level 2 and especially level 1) are challenging. If the superior border of the tumor does not extend beyond the inferior hepatic edge, an abdominal approach is usually used, either by conventional laparotomy or preferably by right subcostal incision [4]. 
If the subcostal approach is used, exposure of the infrahepatic and retrohepatic IVC can be optimized by extending the incision into a lombotomy with the patient in a slightly lateral decubitus position [4]. A thoracoabdominal approach is preferred if the tumor extends to the retrohepatic or suprahepatic part of the IVC. In our case, the excision was comfortably accomplished via a Mercedes-Benz abdominal incision.

Kieffer, et al. mentions of the advantage of combining laparotomy with sternotomy over thoracophrenotomy [4]. With midline incision of the phrenic center, this route gives good exposure of the suprahepatic IVC as well as of the retrohepatic IVC after sectioning of the hepatic ligaments. Sternotomy combined with laparotomy has the added benefit of providing good conditions for establishing cardiopulmonary bypass for treatment of intracardiac extension if present $[4,6]$.

Some authors recommend wide tumor resection at a safe distance from the tumor $[4,8]$. Partial resection of the IVC followed by direct suture or prosthetic patch angioplasty is rarely curable. Complete resection of the IVC is mandated in most cases. In all cases, a frozen section documentation of negative resection margins is desirable (as was done in our case).

Simple ligation is possible after complete or subtotal resection of the infra-renal IVC (Level 3) and/or retrohepatic portion of the IVC (Level 2) in association with resection of the right kidney. There is a difference in collateral circulation between the left and the right. Collaterals on the left (capsular, genital, reno-azygo-lumbar veins) are generally sufficient for satisfactory venous return without occurrence of renal insufficiency. On the contrary, on the right there are no effective collaterals [4].

In cases of incomplete obstruction of the IVC, ligation may be required especially if the procedure involves the gastrointestinal tract, whereby the risk of prosthetic infection is high. Pressure monitoring is performed in such cases to rule out excessive venous hypertension during clamping. As a general rule, a proximal pressure reading of 30 $\mathrm{mm} \mathrm{Hg}$ or more indicates caval reconstruction to avoid postoperative edema of the lower extremity.

Ringed reinforced ePTFE graft is prosthesis of choice for IVC replacement. It provides the best results, given the length of the missing segment and the need for strength to resist compression in the abdomen. Collapse of the graft may be an important factor in thrombosis. Many authors prefer a 20-mm-diameter graft for best congruency with the native vessel $[7,8,10]$. Others recommend smaller grafts $(14-16 \mathrm{~mm})$ for infrarenal replacement to increase blood velocity $[10,15]$. We found a $22 \mathrm{~mm}$ graft best matched the IVC diameter of the patient.

Some authors strongly recommended the creation of an arteriovenous fistula to ensure patency. The fistula eliminates the need for long-term anticoagulation therapy.

For infrahepatic IVC reconstruction simple clamping is often adequate. Complications such as arterial hypotension or proximal venous hypertension are rare and can be dealt with by associated clamping of the infrarenal aorta or supraceliac aorta. Vascular exclusion, indicated for tumors extending to the retrohepatic portion of the IVC allows to perform the upper anastomosis of the prosthesis near the infrahepatic veins so that hepatic circulation can be reestablished quickly thus reducing hepatic ischemia time $[4,11]$. Vascular exclusion also makes it possible to perform associated right or left extended hepatectomy.

For surgical treatment of tumors involving the suprahepatic segment of the IVC venovenous shunting with selective hypothermic hepatic perfusion may be helpful $[4,12,13]$. These modalities provide adequate time cushion for anastomosis of the prosthesis flush with the right atrium and for reimplantation of a patch containing the suprahepatic veins.

Reports of long-term patency have been well documented in literature without anticoagulation although in our case we decided to administer anticoagulation therapy $[10,15]$. Infection of synthetic grafts is a concern. Omental interposition between the graft and resected viscera may be beneficial.

In rare cases involving tumors with intracardiac extension, cardiopulmonary bypass may be required. Venous cannulations should be placed through the tip of the right atrium for the territory drained by the SVC and in the femoral vein or infrarenal IVC for the territory drained by the IVC. Some authors prefer to perform hypothermic circulatory arrest at $18{ }^{\circ} \mathrm{C}$ rather than normothermic cardiopulmonary bypass because the former provides a blood-free operating field.

Right nephrectomy is often required for tumors involving Level 2 of the IVC, even if the kidney is not directly involved. If the tumor only brushes the ostium of the right kidney vein, auto transplantation of the right kidney can be performed into the right iliac fossa. This procedure requires prosthetic reconstruction of the IVC.

Aggressive management of advanced abdominal tumors can produce long-term survival. The 5-year actuarial survival rate after curative resection exceeds $50 \%$ for renal cell carcinoma with caval extension and $28 \%$ for primary leiomyosarcoma $[7,10,15-17]$. On the other hand, median survival without resection is 1 month for patients with primary leiomyosarcoma $[7,15]$.

\section{Adjuvant Therapy}

Chemotherapy, using adriamycin-ifosfamide (as was used in our case) or chemotherapy combined with radiation therapy offer potential adjuvant treatment options [5,18]. The 5-year cumulative survival rate was $53 \%$ for patients with leiomyosarcoma of the IVC suggesting that aggressive surgical management combined with adjuvant therapy offers the best treatment for patients with leiomyosarcoma of the IVC $[5,18]$.

\section{Prognosis}

Even with radical surgery, 5- and 10-year survival of $49.4 \%$ and $29.5 \%$ respectively has been reported [19]. With recurrence rates as high as $50 \%$ reported in some literature, surgery may simply be providing palliation in many cases [19]. Tumors of the retrohepatic IVC tend to present earlier as a result of pressure exerted on surrounding structures. A superior prognosis with 5- and 10-year survival rates of $56.7 \%$ and $47.3 \%$ are reported [19]. Abdominal pain, the clinical presence of a mass and the ability to achieve clear resection margins were noted to be favorable factors whereas poor prognostic indicators include high-grade tumors, suprahepatic tumors, and 
presentation with IVC occlusion or Budd-Chiari syndrome [20].

\section{References}

1. Alexander A, Rehders A, Raffel A, Poremba C, Knoefel WT, Eisenberge $\mathrm{CF}$. Leiomyosarcoma of the inferior vena cava: radical surgery and vascular reconstruction. World J Surg Oncol. 2009; 7: 56.

2. Mingoli A, Cavallaro A, Sapienza P, Di Marzo L, Feldhaus RJ, Cavallari N International registry of inferior vena cava leiomyosarcoma: analysis of a world series on 218 patients. Anticancer Res. 1996; 16: 3201-3205.

3. Hartman DS, Hayes WS, Choyke PL, Tibbetts GP. From the archives of the AFIP. Leiomyosarcoma of the retroperitoneum and inferior vena cava: radiologic-pathologic correlation. Radiographics. 1992; 12: 1203-1220.

4. Kieffer E, Alaoui M, Piette JC, Cacoub P, Chiche L. Leiomyosarcoma of the inferior vena cava: experience in 22 cases. Ann Surg. 2006; 244: 289-295.

5. Huguet C, Ferri M, Gavelli A. Resection of the suprarenal inferior vena cava The role of prosthetic replacement. Arch Surg. 1995; 130: 793-797.

6. Ito H, Hornick JL, Bertagnolli MM, George S, Morgan JA, Baldini EH, et al. Leiomyosarcoma of the inferior vena cava: survival after aggressive management. Ann Surg Oncol. 2007; 14: 3534-3541.

7. Fonesca N, Silvestre I, Bernardino L, et al. Leiomyosarcoma da Veia Cava Inferior-A Proposito de um Caso Clinico Muito Raro. Rev Port Cardiol. 2002; 21: $1496-1478$

8. Hollenbeck ST, Grobmyer SR, Kent KC, Brennan MF. Surgical treatment and outcomes of patients with primary inferior vena cava leiomyosarcoma. J Am Coll Surg. 2003; 197: 575-579.

9. Bower TC, Nagorney DM, Cherry KJ Jr, Toomey BJ, Hallett JW, Panneton $\mathrm{JM}$, et al. Replacement of the inferior vena cava for malignancy: an update. J Vasc Surg. 2000; 31: 270-281.

10. Hines OJ, Nelson S, Quinones-Baldrich WJ, Eilber FR. Leiomyosarcoma of the inferior vena cava: prognosis and comparison with leiomyosarcoma of other anatomic sites. Cancer. 1999; 85: 1077-1083.

11. Hardwigsen J, Baque P, Crespy B, Moutardier V, Delpero JR, Le Treut YP Resection of the inferior vena cava for neoplasms with or without prosthetic replacement: a 14-patient series. Ann Surg. 2001; 233: 242-249.
12. Aller R, Moreira V, Bermejo F, Sanroman AL, de Luis DA. Leiomyosarcoma of the inferior vena cava. The diagnostic and therapeutic approach. Rev Esp Enferm Dig. 1997; 89: 706-710.

13. De San Ildefonso Pereira A, Casal Nunez JE, Maruri Chimeno I, Lojo Sanchez JL, Robledo Gonzalez H, Nuno Vazquez-Garza J. Leiomyosarcoma of the inferior vena cava: therapeutic and clinical considerations. Rev Esp Enferm Dig. 1997; 89: 407-410.

14. Kyriazi MA, Stafyla VK, Chatzinikolaou I, Koureas A, Chatziioannou A, KondiPaphiti A, et al. Surgical challenges in the treatment of leiomyosarcoma of the inferior vena cava: analysis of two cases and brief review of the literature. Ann Vasc Surg. 2010; 24: 826

15. Mingoli A, Feldhaus RJ, Cavallaro A, Stipa S. Leiomyosarcoma of the inferior vena cava: analysis and search of world literature on 141 patients and report of three new cases. J Vasc Surg. 1991; 14: 688-699.

16. Zagars GK, Ballo MT, Pisters PW, Pollock RE, Patel SR, Benjamin RS, et al. Prognostic factors for patients with localized soft-tissue sarcoma treated with conservation surgery and radiation therapy: an analysis of 1225 patients. Cancer. 2003; 97: 2530-2543.

17. Dew J, Hansen K, Hammon J, McCoy T, Levine EA, Shen P. Leiomyosarcoma of the inferior vena cava: surgical management and clinical results. Am Surg 2005; 71: 497-501.

18. Reddy VP, Vanveldhuizen PJ, Muehlebach GF, Dusing RW, Birkbeck JP Williamson SK, et al. Leiomyosarcoma of the inferior vena cava: a case report and review of the literature. Cases J. 2010; 3: 71.

19. Abisi S, Morris-Stiff GJ, Scott-Coombes D, Williams IM, Douglas-Jones AG, Puntis MC. Leiomyosarcoma of the inferior vena cava: clinical experience with four cases. World J Surg Oncol. 2006; 4: 1.

20. Biswas S, Amin A, Chaudry S, Joseph S. Leiomyosarcoma of the inferior vena cava - Radical resection, vascular reconstruction and challenges. World Journal of Oncology. 2013; 4: 107-113
Austin J Clin Case Rep - Volume 4 Issue 2 - 2017

ISSN : 2381-912X | www.austinpublishing group.com

Chaudhary et al. (C) All rights are reserved
Citation: Chaudhary RJ, Rastogi A, Pahari H, Thiagarajan S, Bhangui P, Goja S, et al. Leiomyosarcoma of Intrahepatic IVC - Treatment with Radical Resection and IVC Replacement with PTFE Graft: A Case Report and Review of Literature. Austin J Clin Case Rep. 2017; 4(2): 1118. 